Case Study

\title{
Effects of combined chest expansion and breathing exercises in a patient with sternal pain
}

\author{
WON-GYU YOO ${ }^{1)}$ \\ 1) Department of Physical Therapy, College of Healthcare Medical Science \& Engineering, \\ Inje University: 607 Obangdong, Gimhae, Gyeongsangnam-do 621-749, Republic of Korea
}

\begin{abstract}
Purpose] To investigate the effects of combined chest expansion and breathing exercises in a patient with sternal pain. [Subject and Methods] The study subject was a 36-year-old female employed as a babysitter who complained of sternal pain at the T1-4 level. The subject performed the combined chest expansion and breathing exercises. [Results] The thoracic kyphosis angle after combined chest expansion and breathing exercises decreased compared with the initial angle. The sternal pain after these exercises also decreased compared with the initial value. [Conclusion] This study suggests that combined chest expansion and breathing exercises can be effective in treating sternal pain.

Key words: Breathing exercise, Chest pain, Sternum
\end{abstract}

(This article was submitted May 23, 2017, and was accepted Jun. 12, 2017)

\section{INTRODUCTION}

The sternum is a flat bone that is located in the center of the anterior thoracic wall. It consists of three segments: the manubrium, the body, and the xiphoid process ${ }^{1}$. There are many causes of chest pain (musculoskeletal, gastrointestinal, and cardiac), and it can be frustrating to be in pain ${ }^{2}$. Although rare, stress fractures can be caused by repeated pressure on the sternum, such as through rowing, throwing, wrestling, golfing, bench pressing, or even repeated sit-ups ${ }^{3)}$. Musculoskeletal causes of sternal pain include pectoral muscle ruptures, which generally occur during forceful activities ${ }^{3)}$. The intercostal muscles can also become strained, often as a result of rapid twisting of the torso ${ }^{2}$. After the initial treatment to manage the chest pain, therapy is based on increasing the circulation, collagen production, and extensibility of connective tissues ${ }^{4)}$. In this study, we investigated the effects of combined chest expansion and breathing exercises in a patient with sternal pain.

\section{SUBJECT AND METHODS}

The study subject was a 36-year-old female employed as a babysitter who complained of sternal pain at the T1-4 level and had rounded shoulders and an excessive kyphosis angle. The purpose and methods of the study were explained to the participant, and she provided informed consent. The study adhered to the principles of the Declaration of Helsinki. She had complained of continued upper thoracic pain for 2 months but had not undergone any specific treatment for this condition. She had rounded shoulders and an excessive kyphosis angle. The pain consisted of mechanical sternal pain without radiating pain. She felt sudden intercostal pain after a twisting motion of the trunk. The visual analog scale (VAS) score for this back pain was 7.

A Dual Inclinometer (Acumar, Lafayette Instrument Co., Lafayette, USA) was used to measure the thoracic kyphosis angle. The feet of the inclinometer were placed over the spinal processes thought to correspond to the 1st and 2nd thoracic spines and over the 12th thoracic and 1st lumbar spines. These spinal levels were determined by palpation. Measurements 
were taken with the patient in a natural relaxed, standing posture. The initial thoracic kyphosis angle was $42^{\circ}$.

The subject performed the combined chest expansion and breathing exercises. The combined chest expansion and breathing exercises involved thoracic extension and shoulder retraction with holding of the breath for a few seconds (breathing in slowly and opening her lungs as much as she could). During the thoracic extension exercise, she clasped both arms behind her thorax, gently arching, while flexing the elbows. For 15 days, she performed 10 sets of 10 repetitions of the thoracic extension exercises daily.

\section{RESULTS}

The thoracic kyphosis angle $\left(33^{\circ}\right)$ after combined chest expansion and breathing exercises decreased compared with the initial angle $\left(42^{\circ}\right)$. The sternal pain (VAS 2) after these exercises also decreased compared with the initial value (VAS 7).

\section{DISCUSSION}

A grade 1 (strain) or a grade 2 (partial tear) chest muscle injury can be treated with physical therapy ${ }^{1}$. Thus, excessive exercise will not help in the recovery from strains or partial tears of chest muscles ${ }^{3,4}$. The combined chest expansion and breathing exercises here were mild. The subject did not feel sternal pain during exercise. The forward movement of the front of the body would be expected to further increase the pain. The chest expansion exercise greatly decreased the excessive thoracic kyphosis angle. The expansion of the rib extends to the sternal muscles; indeed, expansion of the rib cage effectively relaxed the sternal muscles. Sternal pain decreased after the combined chest expansion and breathing exercises. In women, changes in the size of the chest can be caused by childbirth and breastfeeding. These changes can cause rounded shoulders and an excess kyphosis angle. Excessive stress in the sternum may also be caused by excessive resistance while carrying a baby. Excessive thoracic kyphosis increases the external moment, inducing curvature in the trunk and compressing the chest $^{1,3}$. Abnormal chest pressure may cause stress in the cartilage between the sternum and ribs. An excessive kyphosis angle can also change the distance between the ribs, which may also cause excessive stress and inflammation in the sternum ${ }^{1)}$. This study suggests that combined chest expansion and breathing exercises can be effective in treating sternal pain.

\section{REFERENCES}

1) Neumann DA: Kinesiology of the musculoskeletal system: foundations for physical rehabilitation. St Louis: Mosby, 2009.

2) Stochkendahl MJ, Christensen HW: Chest pain in focal musculoskeletal disorders. Med Clin North Am, 2010, 94: 259-273. [Medline] [CrossRef]

3) Petilon J, Carr DR, Sekiya JK, et al.: Pectoralis major muscle injuries: evaluation and management. J Am Acad Orthop Surg, 2005, 13: 59-68. [Medline] [CrossRef]

4) Leelarungrayub D, Pothongsunun P, Yankai A, et al.: Acute clinical benefits of chest wall-stretching exercise on expired tidal volume, dyspnea and chest expansion in a patient with chronic obstructive pulmonary disease: a single case study. J Bodyw Mov Ther, 2009, 13: 338-343. [Medline] [CrossRef] 